\title{
Comparative Pharmacokinetic Study of Forchlorfenuron in Adult and Juvenile Rats
}

\author{
Li Ping ${ }^{1}$, Bingyong Xu ${ }^{2}$, Qian Zhou ${ }^{3}$, Yawen Hong ${ }^{1}$, Qingmei Sun ${ }^{1}$, Jincheng Wang ${ }^{1, *}$ and Difeng Zhu ${ }^{1, * \mathbb{D}}$ \\ 1 Center for Drug Safety Evaluation and Research, College of Pharmaceutical Sciences, Zhejiang University, \\ Hangzhou 310058, China; pingli552@zju.edu.cn (L.P.); hongyawen61@zju.edu.cn (Y.H.); \\ 15658223676@163.com (Q.S.) \\ 2 Hangzhou Heze Pharmaceutical Technology Co., Ltd., Hangzhou 310018, China; \\ xubingyong@hezepharm.com \\ 3 Department of Pharmacy, Hangzhou Medical College, Hangzhou 310053, China; qianz1220@hmc.edu.cn \\ * $\quad$ Correspondence: wangjincheng@zju.edu.cn (J.W.); zhudf@zju.edu.cn (D.Z.); Tel.: +86-1538-118-2017 (D.Z.)
}

Citation: Ping, L.; Xu, B.; Zhou, Q.; Hong, Y.; Sun, Q.; Wang, J.; Zhu, D. Comparative Pharmacokinetic Study of Forchlorfenuron in Adult and Juvenile Rats. Molecules 2021, 26, 4276 https://doi.org/10.3390/

molecules 26144276

Academic Editor: Andreas Tzakos

Received: 3 June 2021

Accepted: 13 July 2021

Published: 14 July 2021

Publisher's Note: MDPI stays neutral with regard to jurisdictional claims in published maps and institutional affiliations.

Copyright: (c) 2021 by the authors. Licensee MDPI, Basel, Switzerland. This article is an open access article distributed under the terms and conditions of the Creative Commons Attribution (CC BY) license (https:// creativecommons.org/licenses/by/ $4.0 /)$.

\begin{abstract}
Forchlorfenuron (CPPU) is a plant growth regulator extensively used in agriculture. However, studies on CPPU pharmacokinetics are lacking. We established and validated a rapid, sensitive, and accurate liquid chromatography-mass spectrometry method for CPPU detection in rat plasma. CPPU pharmacokinetics was evaluated in adult and juvenile rats orally treated with 10, 30, and $90 \mathrm{mg} / \mathrm{kg}$ of the compound. The area under the plasma drug concentration-time curve from 0 to $24 \mathrm{~h}$ (AUC), at the final time point sampled $\left(\mathrm{AUC}_{0-\mathrm{t}}\right)$, and the maximum drug concentration of $\mathrm{CPPU}$ increased in a dose-dependent manner. The pharmacokinetic parameters $\mathrm{AUC}_{0-\mathrm{t}}$ and absolute bioavailability were higher in the juvenile rats than in adult rats. The mean residence time and $\mathrm{AUC}_{0-\mathrm{t}}$ of juvenile rats in the gavage groups, except for the $10 \mathrm{mg} / \mathrm{kg}$ dose, were significantly higher in comparison to those observed for adult rats $(p<0.001)$. The plasma clearance of CPPU in juvenile rats was slightly lower than that in the adult rats. Taken together, juvenile rats were more sensitive to CPPU than adult rats, which indicates potential safety risks of CPPU in minors.
\end{abstract}

Keywords: pharmacokinetics; forchlorfenuron; juvenile rat; liquid chromatography-mass spectrometry

\section{Introduction}

Forchlorfenuron, also known as 1-(2-chloro-4-pyridyl)-3-phenylurea (CPPU), is a synthetic phenyl urea-derived cytokinin extensively used as a plant growth regulator in agriculture. Its major effects include the promotion of cell division, growth, and differentiation. CPPU has already been registered for use in many countries, including European Union countries, the USA, and China [1], and is widely applied in kiwifruit, watermelon, cucumber, and grape cultivation [2]. However, the excessive and inappropriate use of CPPU in recent years has led to concerns regarding its safety [3].

Previous research has reported CPPU-associated toxicity. Gong et al. found that CPPU could induce cardiotoxicity, including cardiac deformation, cardiac contractile dysfunction, and anemia, in zebrafish [4]. An in vitro study demonstrated that CPPU was cytotoxic to normal Chinese hamster ovary cells, with an $\mathrm{IC}_{50}$ value of $12.1 \pm 2.1 \mu \mathrm{M}$ [5]. A study by Qian et al. showed that CPPU down-regulated the expression of CYP17A1 and CYP19A1, and, consequently, estradiol and progesterone production in Sprague-Dawley rats [6], while also suggesting that it may promote estradiol secretion. Adverse effects have been reported in prepubertal female rats [7], indicative of reproductive toxicity following CPPU exposure. CYP17A1 and CYP19A1 are also expressed in humans, with CYP17A1 playing a key role in steroid hormone synthesis and CYP19A1 being the rate-limiting enzyme of estrogen synthesis from androgen [8,9].

A previous study demonstrated that juvenile animals are more susceptible to the acute effects of some organophosphorus insecticides than adults [10]. Based on these 
reports, pesticide exposure is expected to induce a more toxic response in children. The risk of pesticide exposure in children has received widespread attention in the field of public health. In this context, children should not be considered small adults, but rather a unique subpopulation with an altered vulnerability to chemical insults due to differences in physiological and metabolic functions [11,12].

CPPU residues have been reported in fruit $[2,13]$, suggesting that humans may be exposed to CPPU through the intake of both fruit and vegetables [14,15]. While CPPU ingestion may have detrimental health effects, studies on its pharmacokinetics (PK) in animals have been limited, it is necessary to develop a method for the detection of plasma CPPU. The PK characteristics of CPPU in children are different from those in adults, as the former are more sensitive to chemical compounds [12,16-18]. While the PK characteristics of CPPU in juvenile rats are currently unclear, understanding these may provide a basis for the further study of pesticide exposure in children. Therefore, it is important to explore the comparative PK characteristics of CPPU in juvenile versus adult rats.

In this study, we developed and validated a rapid liquid chromatography-mass spectrometry (LC-MS/MS) method to examine CPPU concentration in rat plasma. We applied the method to compare the PK characteristics of CPPU in adult and juvenile rats. Our findings provide evidence for the risks of CPPU exposure in children as well as a scientific basis for the understanding of its metabolism in humans.

\section{Results and Discussion}

\subsection{Optimization of Sample Preparation}

To remove proteins and other components that could potentially cause interference, protein precipitation and liquid-liquid extraction methods were performed, while keeping in mind the chemical properties of CPPU. Both methods indicated that direct protein precipitation with methanol produced a better separation and extraction recovery rate. Thus, direct protein precipitation with methanol was selected for sample preparation.

\subsection{Optimization of LC and MS Conditions}

To optimize the conditions for the detection of CPPU and curcumin, the internal standard (IS), different chromatography and mass spectrometry conditions were evaluated. We compared the results obtained using Xbridge ${ }^{\mathrm{TM}} \mathrm{C} 18(2.1 \mathrm{~mm} \times 50 \mathrm{~mm}, 3.5 \mu \mathrm{m}$; Waters, Milford, MA, USA) column and the ACQUITYUPLCBEHC18 $(2.1 \mathrm{~mm} \times 50 \mathrm{~mm}, 1.7 \mu \mathrm{m}$; Waters) column. The latter exhibited a better resolution and peak shape of the analyte and was therefore chosen for further analysis. Acetonitrile/water and methanol/water binary solvent system using different buffers, such as ammonium formate and formic acid, were tested for the mobile phase. Acetonitrile/water containing $0.1 \%$ formic acid was finally selected, where gradient elution could improve peak shape symmetry and enhance the signal. Finally, we examined flow rates in the range of $0.2-0.5 \mathrm{~mL} / \mathrm{min}$ and found that $0.3 \mathrm{~mL} / \mathrm{min}$ was the best condition.

Electrospray ionization (ESI) was used to generate ions due to its high sensitivity and fragmentation. To optimize the ESI conditions for detecting CPPU and IS, positive $(\mathrm{ESI}+)$ and negative (ESI-) ion detection modes were explored. Both compounds exhibited good responses in the ESI+ detection mode, with a low background noise level. Thus, detection was operated in ESI+ mode. Next, autotune was used to obtain the initial optimal MS parameters; however, the sensitivity for the analyte was not sufficient. Finally, optimal MS conditions, including collision energy, cone voltage, ion source temperature, and desolvation gas temperature, were further optimized via manual manipulation in fine-tuning mode. Representative CPPU LC/MS-MS chromatograms are shown in Figure 1. There was no distinct interference from endogenous peaks under the chromatographic conditions, and the retention times of CPPU and IS were 1.74 and $1.91 \mathrm{~min}$, respectively. These results indicate that the method is specific for the analysis of CPPU in rat plasma. 

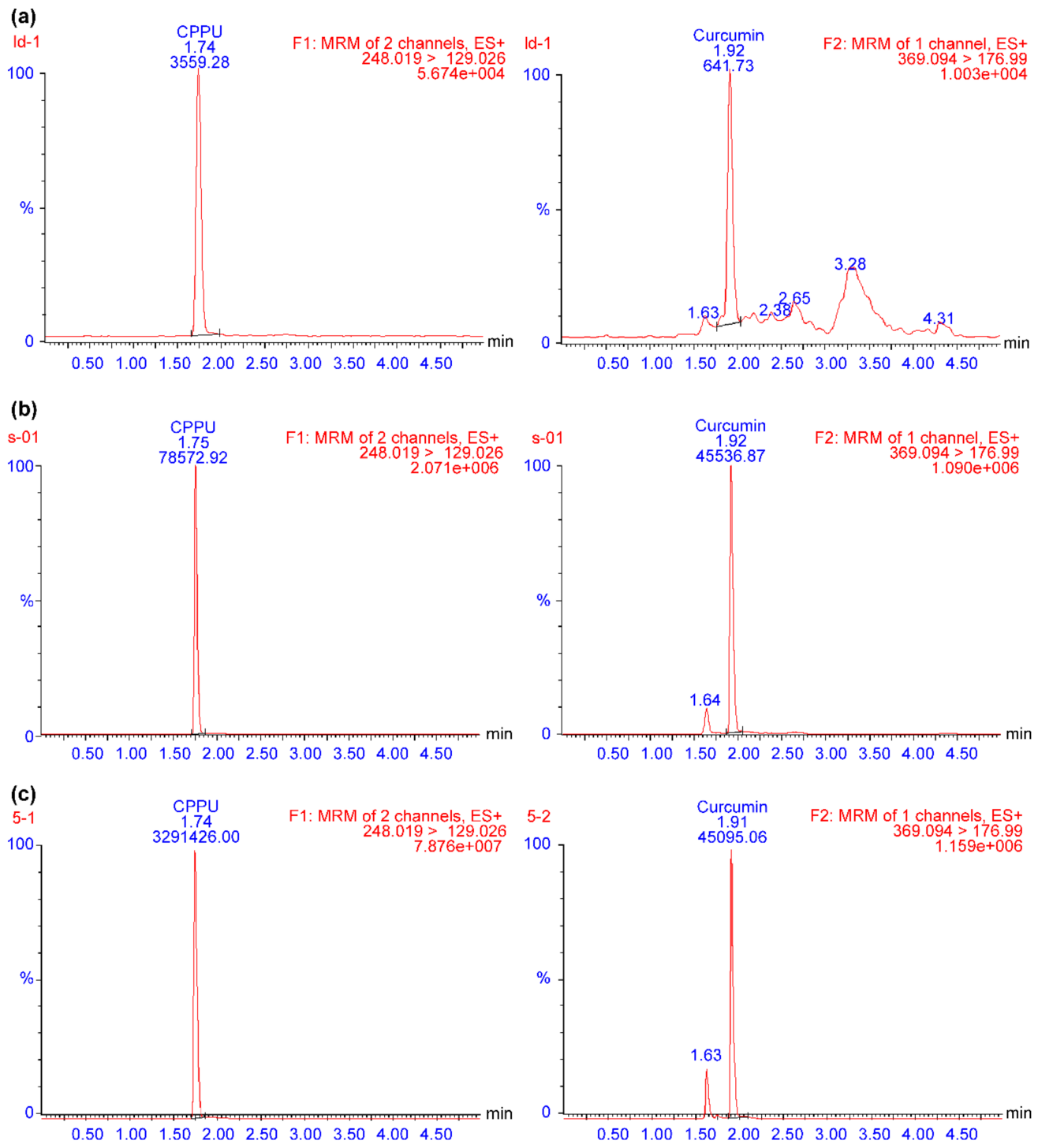

Figure 1. Typical LC/MS-MS chromatograms of (a) blank plasma, (b) blank plasma spiked with CPPU at the LLOQ, and (c) actual sample obtained from a rat $0.5 \mathrm{~h}$ after intragastric administration of CPPU. CPPU-1-(2-chloro-4-pyridyl)-3-phenylurea, forchlorfenuron; LC/MS-MS—liquid chromatography-mass spectrometry; LLOQ—lower limit of quantification.

\subsection{Method Validation}

The CPPU calibration plots were linear in the range of $20-2000 \mathrm{ng} / \mathrm{mL}$ using $1 / \mathrm{x}^{2}$ weighted regression. The representative standard curve equation was

$$
y=0.024793 x+0.0456153
$$

and the correlation co-efficient $(\mathrm{r})$ was $\geq 0.99(\mathrm{r}=0.9988, n=7)$.

The analyte response at the lower limit of quantification (LLOQ) was $20 \mathrm{ng} / \mathrm{mL}$ (\%RSD $=6.5 \%, n=5, \mathrm{~S} / \mathrm{N}>10)$, and the lower limit of detection (LLOD) was $10 \mathrm{ng} / \mathrm{mL}(n=5$, $\mathrm{S} / \mathrm{N}>3)$.

The inter- and intra-assay accuracy was $92.5-102.9 \%$ and $92.2-101.5 \%$, respectively. The intra- and inter-assay precision was $0.8-6.5 \%$ and $0.4-5.4 \%$, respectively. These results met the precision requirement of up to $15 \%$, except for the LLOQ, where a value of $\leq 20 \%$ was observed. The accuracy was within the range of $85-115 \%$, and the LLOQ would include the range of $80-120 \%$ (Table 1). 
Pre-and post-extracted plasma standards containing 60, 1000, and $1600 \mathrm{ng} / \mathrm{mL}$ CPPU were analyzed, and the recovery was $82.5-93.1 \%$. The absolute recovery of $200 \mathrm{ng} / \mathrm{mL}$ IS was $106.5 \%$ (Table 2). The peak area ratios of CPPU with the post-extraction blank sample to CPPU in the mobile phase verified the absence of significant matrix effects (MEs) in our method (Table 2).

Table 1. Inter- and intra-assay accuracy and precision of CPPU detection in rat plasma $(n=5)$.

\begin{tabular}{ccccccc}
\hline \multirow{2}{*}{$\begin{array}{c}\text { Concentration of CPPU } \\
\text { (ng/mL) }\end{array}$} & \multicolumn{3}{c}{ Intra-Assay } & \multicolumn{3}{c}{ Inter-Assay } \\
\cline { 2 - 7 } & Mean \pm SD & Precision (\%) & Accuracy (\%) & Mean \pm SD & Precision (\%) & Accuracy (\%) \\
\hline 20 & $20.6 \pm 1.3$ & 6.5 & 102.9 & $20.2 \pm 1.1$ & 5.4 & 101.2 \\
60 & $61.4 \pm 1.5$ & 2.5 & 102.3 & $60.9 \pm 0.5$ & 0.9 & 101.5 \\
1000 & $933.3 \pm 18$ & 1.9 & 93.3 & $952.8 \pm 17.4$ & 1.8 & 95.3 \\
1600 & $1478.9 \pm 12.2$ & 0.8 & 92.5 & $1475.5 \pm 6.4$ & 0.4 & 92.2 \\
\hline
\end{tabular}

CPPU, 1-(2-chloro-4-pyridyl)-3-phenylurea, forchlorfenuron.

Table 2. Matrix effect and recovery of CPPU and IS $(n=6)$.

\begin{tabular}{cccc}
\hline Substance & Concentration of CPPU $(\mathbf{n g} / \mathbf{m L})$ & RE (\%) & ME (\%) \\
\hline \multirow{2}{*}{ CPPU } & 60 & $85.7 \pm 0.4$ & $90.5 \pm 1.2$ \\
\cline { 2 - 4 } & 1000 & $82.5 \pm 4.9$ & $98.1 \pm 3.2$ \\
\cline { 2 - 4 } & 1600 & $93.1 \pm 1.3$ & $96.4 \pm 1.1$ \\
\hline IS & 200 & $106.5 \pm 5.3$ & $108.3 \pm 5.2$ \\
\hline ME—-matrix effect; RE-recovery; IS—internal standard (curcumin); CPPU—-1-(2-chloro-4-pyridyl)-3-phenylurea,
\end{tabular}
forchlorfenuron.

CPPU samples were stable after storage at room temperature $\left(20-24^{\circ} \mathrm{C}\right)$ for $12 \mathrm{~h}$, at $-20{ }^{\circ} \mathrm{C}$ for 14 days, after at least three freeze-thaw cycles at $-20^{\circ} \mathrm{C}$, and in the sample rack $\left(4^{\circ} \mathrm{C}\right)$ for $24 \mathrm{~h}$ (Table 3$)$.

Table 3. Stability of CPPU $(n=3)$.

\begin{tabular}{|c|c|c|c|c|c|c|c|c|}
\hline \multirow{2}{*}{$\begin{array}{l}\text { Spiked Samples } \\
\text { (ng/mL) }\end{array}$} & \multicolumn{2}{|c|}{$12 \mathrm{~h}$ (Benchtop) } & \multicolumn{2}{|c|}{24 h (Autosampler) } & \multicolumn{2}{|c|}{14 Days (at $-20^{\circ} \mathrm{C}$ ) } & \multicolumn{2}{|c|}{ Three Freeze-Thaw Cycles } \\
\hline & Mean \pm SD & Accuracy (\%) & Mean \pm SD & Accuracy (\%) & Mean \pm SD & Accuracy $(\%)$ & Mean \pm SD & Accuracy (\%) \\
\hline 60 & $57.5 \pm 1.7$ & 95.9 & $58.5 \pm 1.3$ & 97.5 & $61.6 \pm 0.9$ & 102.7 & $61.4 \pm 1.6$ & 101.7 \\
\hline 1000 & $974.6 \pm 16.7$ & 97.5 & $970.6 \pm 8.4$ & 97.1 & $1042.7 \pm 23.1$ & 104.3 & $1030.7 \pm 12.1$ & 103.1 \\
\hline 1600 & $\begin{array}{c}1564.9 \pm \\
23.7\end{array}$ & 97.8 & $\begin{array}{c}1546.3 \pm \\
27.3\end{array}$ & 96.6 & $1664.4 \pm 17.5$ & 104.0 & $1632.9 \pm 8.9$ & 102.1 \\
\hline
\end{tabular}

CPPU-1-(2-chloro-4-pyridyl)-3-phenylurea, forchlorfenuron.

The accuracy and precision of CPPU detection under the corresponding dilution factors were within $\pm 15 \%$, indicating that the plasma samples were stable under the $10 \times$ dilution conditions. The residue was determined by measuring the blank plasma sample after detecting the upper limit of the standard curve, and the residual response measured in the blank sample was not more than $20 \%$ of the LLOQ, indicating that the residue met the requirements.

\subsection{Application in a Comparative PK Study of Rats}

In this study, a rapid, simple, and efficient method was developed for the determination of CPPU in rat plasma. Using this method, we performed a comparative PK study of CPPU in adult and juvenile rats. The mean plasma concentration-time curves obtained after the oral administration of CPPU to adult and juvenile rats are shown in Figure 2. Using DAS v.3.1 software, the non-compartment model was found to be the most rational PK model in both adult and juvenile rats. The correlation between $\mathrm{AUC}_{0-\mathrm{t}}$ and dose is shown in Figure 3. The CPPU did not induce toxicity in rats at the administered doses, and the vital signs of the rats were normal. The PK parameters are shown in Table 4. 

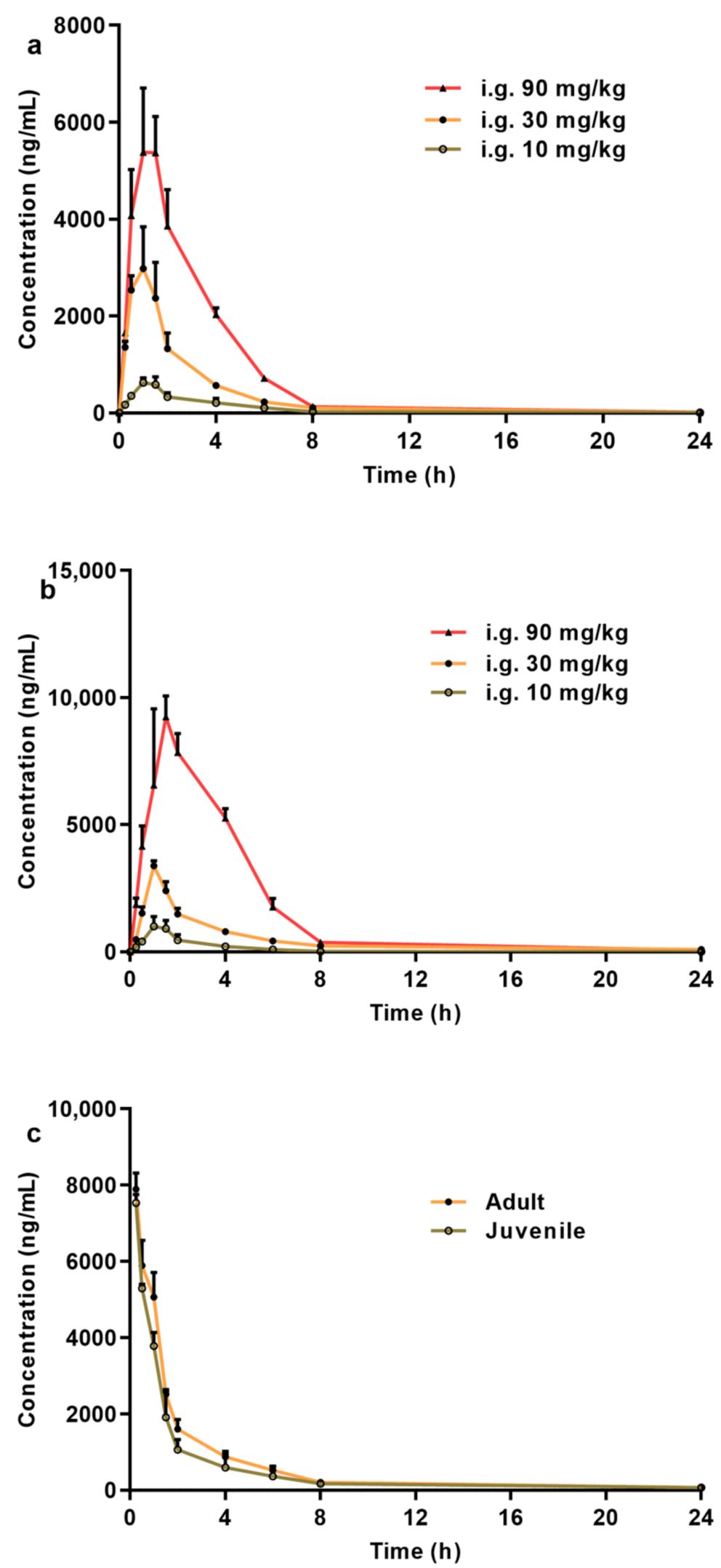

Figure 2. (a) Mean plasma concentration-time curves following the oral administration of CPPU in adult rats. (b) Mean plasma concentration-time curves following oral administration of CPPU in juvenile rats. (c) Mean plasma concentration-time curves following intravenous administration of CPPU in both adult and juvenile rats. CPPU-1-(2-chloro-4-pyridyl)-3-phenylurea, forchlorfenuron. 


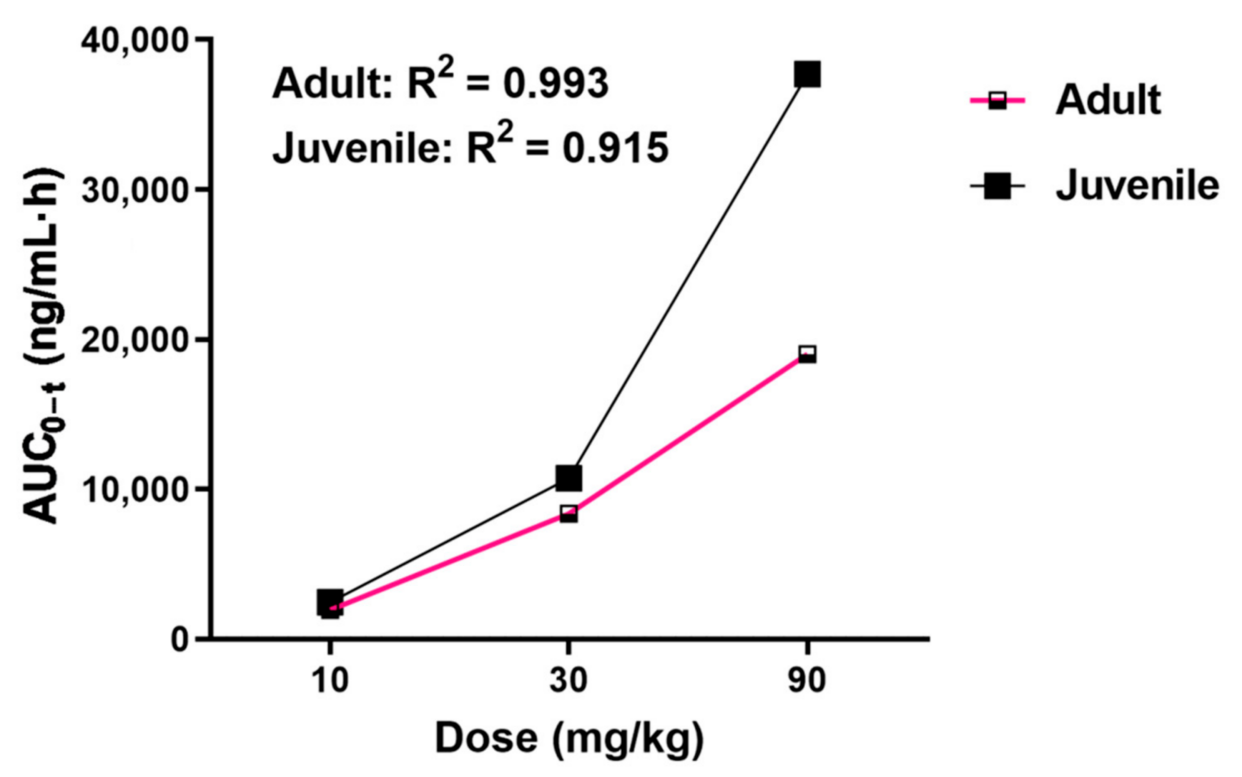

Figure 3. Area under the concentration-time curve from time " 0 " to the time of the last measurable concentration $\left(\mathrm{AUC}_{0-\mathrm{t}}\right)$ vs. CPPU dose: comparison between adult and juvenile rats. CPPU-1-(2chloro-4-pyridyl)-3-phenylurea, forchlorfenuron.

Table 4. Pharmacokinetic (PK) parameters of CPPU.

\begin{tabular}{|c|c|c|c|c|c|c|c|c|}
\hline Animals & Dose (mg/kg) & $\mathrm{C}_{\max }(\mathrm{ng} / \mathrm{mL})$ & $\mathrm{T}_{1 / 2}(\mathrm{~h})$ & CLz (L/h/kg) & $\mathrm{MRT}_{0-\mathrm{t}}(\mathrm{h})$ & $\mathrm{AUC}_{0-\mathrm{t}}(\mathrm{ng} / \mathrm{mL} \cdot \mathrm{h})$ & $\mathrm{AUC}_{0-\infty}(\mathrm{ng} / \mathrm{mL} \cdot \mathrm{h})$ & $F(\%)$ \\
\hline \multirow{4}{*}{ Adult rats } & 10 (p.o.) & $711.1 \pm 70.4$ & $2.6 \pm 1.1$ & $5.0 \pm 1.3$ & $3.0 \pm 0.6$ & $1935.7 \pm 361.5$ & $2108.3 \pm 553.3$ & 6.1 \\
\hline & 30 (p.o.) & $3266.3 \pm 50.2$ & $2.4 \pm 1.1$ & $3.4 \pm 0.7$ & $3.1 \pm 0.6$ & $9294.4 \pm 862.0$ & $9310.6 \pm 867.7$ & 9.8 \\
\hline & 90 (p.o.) & $6273.3 \pm 133.1$ & $2.5 \pm 0.8$ & $4.6 \pm 0.2$ & $2.9 \pm 0.1$ & $18,915.9 \pm 622.7$ & $18,967.7 \pm 643.2$ & 6.6 \\
\hline & 5 (i.v.) & $7977.4 \pm 143.7$ & $1.8 \pm 0.1$ & $0.3 \pm 0.01$ & $3.0 \pm 0.1$ & $15,851.6 \pm 351.9$ & $15,852.4 \pm 352.8$ & - \\
\hline \multirow{4}{*}{ Juvenile rats } & 10 (p.o.) & $1148.9 \pm 145.1^{* *}$ & $2.6 \pm 1.1$ & $3.8 \pm 0.9$ & $2.6 \pm 0.3$ & $2745.5 \pm 632.7$ & $2753.6 \pm 608.1$ & 10.2 \\
\hline & 30 (p.o.) & $3386.1 \pm 195.5$ & $2.3 \pm 0.2$ & $2.8 \pm 0.2$ & $4.9 \pm 0.1^{* * *}$ & $10,713.5 \pm 559.5^{* * *}$ & $10,720.4 \pm 563.4^{* * *}$ & 13.3 \\
\hline & 90 (p.o.) & $9805.7 \pm 77.5^{* * *}$ & $2.5 \pm 0.1$ & $2.5 \pm 0.1$ & $3.3 \pm 0.1^{* * *}$ & $36,442.7 \pm 1086.1^{* * *}$ & $36,513.9 \pm 1085.8^{* * *}$ & 15.1 \\
\hline & 5 (i.v.) & $7571.2 \pm 183.1$ & $1.7 \pm 0.4$ & $0.3 \pm 0.04$ & $2.9 \pm 0.3$ & $13,470.0 \pm 1281.6$ & $13,471.3 \pm 1280.4$ & - \\
\hline
\end{tabular}

Data are presented as the mean $\pm \mathrm{SD}(n=6)$. i.v.-intravenous; p.o.-per oral; $\mathrm{C}_{\max }$ - the maximum drug concentration; $\mathrm{T}_{1 / 2}-$ the time taken for half the initial drug concentration to be eliminated; CLz-plasma clearance; $\mathrm{MRT}_{0-\mathrm{t}}-\mathrm{mean}_{\mathrm{m}}$ residence time; $\mathrm{AUC}_{0-\mathrm{t}}-$ the area under the plasma drug concentration-time curve from 0 to $24 \mathrm{~h}$, the final time point sampled; $\mathrm{AUC}_{0-\infty}-$ the area $_{\mathrm{under}}$ the plasma drug concentration-time curve from 0 to infinity; comparison between the doses in adult and juvenile rats; F-bioavailability, CPPU-1-(2-chloro-4-pyridyl)-3-phenylurea, forchlorfenuron. ${ }^{* *} p<0.01$ and ${ }^{* * *} p<0.001$.

Both the $\mathrm{AUC}_{0-\mathrm{t}}$ and maximum drug concentration $\left(\mathrm{C}_{\max }\right)$ in adult and juvenile rats treated with CPPU at $10-90 \mathrm{mg} / \mathrm{kg}$ increased in a dose-dependent manner. The $\mathrm{AUC}_{0-\mathrm{t}}$ and $F$ values in the juvenile rats were higher than those in the adult rats. The $T_{1 / 2}$ in adult rats was similar to that in the juvenile rats. The mean residence time $\left(\mathrm{MRT}_{0-\mathrm{t}}\right)$ and $\mathrm{AUC}_{0-\mathrm{t}}$ of juvenile rats in the gavage groups, except for the $10 \mathrm{mg} / \mathrm{kg}$ dose, were higher than those in adult rats $(p<0.001)$. Although the $C_{\max }$ of the $30 \mathrm{mg} / \mathrm{kg}$ dose group (juvenile rats) increased slightly, the $C_{\max }$ of the 10 and $90 \mathrm{mg} / \mathrm{kg}$ groups showed a significant increase. The plasma clearance (CLz) of CPPU in juvenile rats was slightly lower than that in adult rats. These results indicate that juvenile rats are more sensitive to CPPU.

According to the toxicology data issued by the United States Environmental Protection Agency (US EPA [19]), chronic and sub-chronic studies have indicated that CPPU is toxic to developing zebrafish, as determined during screening for developmental toxicity, with a half-maximal activity of $46 \mu \mathrm{M}$ [20]. Another study found that the administration of high-dose CPPU to rats (115 mg/ $\mathrm{kg}$ for males and $205 \mathrm{mg} / \mathrm{kg}$ for females) caused slight decreases in food intake and body weight of first (F1) and second (F2) filial generation animals [21]. Zhu et al. conducted a study on the developmental toxicity of CPPU administered to rats via gavage prenatally and postnatally. They found that CPPU may 
promote estradiol secretion, resulting in altered vaginal opening and first estrus time in prepubertal female rats [3]. These studies suggest that CPPU has potent effects on animal reproduction and development. While a few analytical methods for CPPU detection, such as high-performance liquid chromatography (HPLC) with ultraviolet detection [22], LCMS/MS [13,22], and LC/time-of-flight/MS [23,24], few reports evaluating the PK of CPPU were published to date. We studied the PK characteristics of CPPU in adult and juvenile rats and found that $\mathrm{AUC}_{0-\mathrm{t}}$ and absolute bioavailability were higher in juvenile than in adult rats, which may be due to metabolic differences.

\section{Materials and Methods}

\subsection{Chemicals and Reagents}

CPPU (99\% purity) was provided by Bio Basic Canada (Markham, Ont., Canada). Curcumin (IS; $98.7 \%$ purity) was purchased from the National Institutes for Food and Drug Control (Beijing, China). The structures of CPPU and IS are shown in Figure 4. Acetonitrile, methanol, and formic acid were provided by Merck (Darmstadt, Germany). All solvents were of chromatography grade. Purified water was purchased from ELGAPURELAB Ultra System (High Wycombe, UK).<smiles>O=C(Nc1ccccc1)Nc1ccnc(Cl)c1</smiles>

CPPU<smiles>COc1cc(/C=C/C(=O)CC(=O)/C=C/c2ccc(O)c(OC)c2)ccc1O</smiles>

Curcumin

Figure 4. Structural formulas of 1-(2-chloro-4-pyridyl)-3-phenylurea (CPPU, forchlorfenuron), and curcumin.

\subsection{Animals}

Adult (6- to 8-week-old, weight: 200-220 g) and juvenile (3- to 4-week-old, weight: 80-100 g) Sprague-Dawley rats (equal numbers of males and females) were obtained from Vital River Laboratory Animal Technology Co. (Beijing, China). The animals were housed at an ambient temperature of $20-26^{\circ} \mathrm{C}$ and humidity of $40-70 \%$. The pressure between the room and corridor was greater than $10 \mathrm{~Pa}$, with a ventilation over 15 times per hour, and a 12/12-h light/dark cycle (08:00-20:00). The animals were acclimatized to these conditions for one week and were fasted for at least $12 \mathrm{~h}$ before the experiments. The study was approved by the Institutional Animal Care and Use Committee of CDSER of Zhejiang University (IACUC-17-211).

\subsection{Instrumentation and Chromatographic Conditions}

A triple-quadrupole ultra-high-performance liquid chromatography (UPLC) system (Xevo, Waters Corp., Milford, MA, USA) was used for CPPU analysis. Considering the properties of CPPU and large sample volumes, various UPLC columns with different pore diameters were investigated. Based on our optimization experiments, ACQUITY UPLC BEHC18 column $(2.1 \mathrm{~mm} \times 50 \mathrm{~mm}, 1.7 \mu \mathrm{m}$, Waters $)$ was selected and the analysis temperature was set at $30{ }^{\circ} \mathrm{C}$. Acetonitrile/water and methanol/water binary solvent systems using different buffers, such as ammonium formate and formic acid, were tested; acetonitrile (B) and water containing $0.1 \%$ formic acid (B) was eventually selected as the mobile phase at a flow rate of $0.3 \mathrm{~mL} / \mathrm{min}$. Gradient elution was performed as follows: 0-2 $\min , 10-95 \%$ B; 2-3.5 $\mathrm{min}, 95 \% \mathrm{~B}$; 3.5-4 $\mathrm{min}, 95-10 \% \mathrm{~B}$; and $4-5 \mathrm{~min}, 10 \% \mathrm{~B}$.

CPPU and curcumin were detected using the positive multiple reaction monitoring mode. CPPU ions were detected at $m / z 248.02 \rightarrow 93.07$, and curcumin ions were detected at $m / z 369.09 \rightarrow 176.99$. The MS operating parameters were optimized as follows: source temperature, $150{ }^{\circ} \mathrm{C}$; desolvation temperature, $500{ }^{\circ} \mathrm{C}$; capillary voltage, $3 \mathrm{kV}$; collision gas 
flow, $0.15 \mathrm{~mL} / \mathrm{min}$; cone gas flow, $150 \mathrm{~L} / \mathrm{h}$; desolvation gas (nitrogen) flow, $650 \mathrm{~L} / \mathrm{h}$; and collision energy, 36 and $22 \mathrm{~V}$ for CPPU and curcumin, respectively. Data processing was performed using the MassLynx v.4.1 software (Waters Corp).

\subsection{Preparation of Standard and Quality Control Samples}

Stock solutions of CPPU $(1 \mathrm{mg} / \mathrm{mL})$ and curcumin $(1 \mathrm{mg} / \mathrm{mL})$ were separately prepared in methanol. Calibration standard samples for CPPU were diluted with methanol to obtain concentrations of $40,20,10,4,2,1$, and $0.4 \mu \mathrm{g} / \mathrm{mL}$. Curcumin standard solution was prepared at $200 \mathrm{ng} / \mathrm{mL}$ with methanol. All standard solutions were kept at $-20^{\circ} \mathrm{C}$ until use.

Each blank rat plasma sample $(95 \mu \mathrm{L})$ was spiked with different concentrations of CPPU $(5 \mu \mathrm{L})$ and divided into two parts after mixing. One part $(50 \mu \mathrm{L})$ was used for the calibration standard samples at concentrations of 20,50, 100, 200, 500, 1000, and $2000 \mathrm{ng} / \mathrm{mL}$. The other was kept as a spare portion. The quality control CPPU samples (20, 60,1000 , and $1600 \mathrm{ng} / \mathrm{mL}$ ) were prepared in an identical manner.

\subsection{Sample Preparation}

Methanol precipitation and ethyl acetate liquid-liquid extraction methods were investigated based on the compound properties. Subsequently, methanol precipitation was selected, and a good extraction recovery rate was achieved. Proteins in rat plasma samples were precipitated using three volumes of methanol. Thereafter, $50 \mu \mathrm{L}$ of plasma was pretreated in the same way (three volumes of methanol, in addition to curcumin), vortexed for $10 \mathrm{~min}$, and centrifuged at $12,000 \times g$ for $10 \mathrm{~min}$. The supernatant $(10 \mu \mathrm{L})$ was analyzed using LC-MS/MS. The samples for method validation were pretreated in the same way (three volumes of methanol, in addition to curcumin).

\subsection{Method Validation}

Method validation was conducted following the Bioanalytical Method Validation Guidance for Industry [24]. The following items were investigated: specificity, linearity, precision and accuracy, $\mathrm{LLOQ}$, recovery rate, $\mathrm{ME}$, and dilution reliability.

The chromatograms of six individual sources of blanks were applied to assess specificity. The method was considered specific if the response attributable to interfering components was not more than $20 \%$ of the analyte response at the LLOQ and not more than $5 \%$ of the IS response in the LLOQ sample.

Plasma samples of the standard curve were spiked using seven CPPU concentrations $(20,50,100,200,500,1000$, and $2000 \mathrm{ng} / \mathrm{mL})$. The peak area ratio of CPPU to curcumin was calculated and the standard curve was drawn by plotting the peak area ratio vs. analyte concentration. LLOQ was determined as the minimum concentration of the calibration curve. A signal-to-noise ratio of 3 was used to determine LLOD.

Accuracy and precision were evaluated based on the performance of Quality Control (QC) samples within each run and between different runs. Three batches were continuously detected to investigate the intra-batch and inter-batch precision. The intra-batch and interbatch precision must be $<15 \%$, with an accuracy ranging between $85 \%$ and $115 \%$, except for LLOQ (no more than $20 \%$ or within the range of $80-120 \%$ ).

Recovery was computed by comparing the peak area ratios of QC samples (at different concentrations) with those of spiked, post-extraction blank samples. The acceptance criterion was a $\leq 30 \%$ difference between the $Q C$ concentration recoveries.

The ME was investigated by contrasting the peak responses of pure solution samples at three QC concentrations of CPPU and at a single concentration of IS. The acceptance criterion was set at no more than 15\% for \%RSD.

Sample stability was determined at three concentrations $(60,1000$, and $1600 \mathrm{ng} / \mathrm{mL}$, $n=3$ per concentration) under different test conditions: (1) short-term stability at room temperature $\left(15-30{ }^{\circ} \mathrm{C}\right)$ for $12 \mathrm{~h}$; (2) long-term stability at $-20^{\circ} \mathrm{C}$ for 14 days; (3) post- 
preparation stability in the autosampler at $4^{\circ} \mathrm{C}$ for $24 \mathrm{~h}$; and (4) after three freeze-thaw cycles at $-20^{\circ} \mathrm{C}$.

Some plasma sample concentrations were out of range of the standard curve during sample detection. These samples were diluted and retested. The dilution factor of CPPU was set to 10 according to the linear range. The accuracy and precision were supposed to be within $15 \%$.

For residue verification, residues were determined by examining the upper limit of the standard curves and blank plasma samples. The responses measured using the blank samples were supposed to be $<20 \%$ of the LLOQ.

\subsection{PK Study}

The national long-term dietary estimated daily intake safety evaluation study of the Australian Bureau of Chemical Safety showed that the no observed effect level (NOEL) of CPPU is $7 \mathrm{mg} / \mathrm{kg} /$ person/day [25]. According to the conversion of animal and human doses, the NOEL of rats is therefore $\sim 40 \mathrm{mg} / \mathrm{kg}$. The CPPU doses for rats were consequently set to 10,30 , and $90 \mathrm{mg} / \mathrm{kg}$. Adult rats ( $n=24 ; n=6$ per group) were fasted for at least $12 \mathrm{~h}$ and randomly divided into three treatment groups for oral administration of 10,30 , or $90 \mathrm{mg} / \mathrm{kg}$ CPPU (in 0.5\% carboxymethyl cellulose), and another treatment group received intravenous administration of $5 \mathrm{mg} / \mathrm{kg}$ CPPU (in saline). The juvenile rats $(n=24 ; n=6$ per group) were also randomly divided into four groups following the same dosing regimen. CPPU was suspended in $0.5 \%$ carboxymethyl cellulose at 1,3 , and $9 \mathrm{mg} / \mathrm{mL}$ for oral administration and in saline at $0.5 \mathrm{mg} / \mathrm{mL}$ for intravenous administration. Approximately $100 \mu \mathrm{L}$ blood samples were withdrawn into heparinized tubes using the tail bleed method before the administration as well as at $0.25,0.5,1,1.5,2,4,6,8$, and $24 \mathrm{~h}$ following administration. The blood samples were immediately centrifuged at $1000 \times \mathrm{g}$ for $10 \mathrm{~min}$, and the plasma samples were frozen at $-20^{\circ} \mathrm{C}$ until analysis.

The animal studies were approved by the Animal Research Committee at Zhejiang University (Approval number IACUC-17-211), and all experimental protocols were conducted in accordance with the institutional guidelines. DAS v.3.1 software (DAS, Shanghai, China) was used to calculate and analyze the PK parameters such as $\mathrm{AUC}_{0-\mathrm{t}}, \mathrm{C}_{\max }, \mathrm{T}_{1 / 2}$, MRT, and CL. SPSS Statistics v19.0 (IBM, Armonk, NY, USA) was used to perform statistical tests for the data comparisons. A test for homogeneity of variance was performed. If the variance was uniform $(p>0.05)$, a one-way analysis of variance was performed. If the variance was not uniform $(p<0.05)$, a non-parametric test (Kruskal-Wallis H test) was performed. The summary table report lists the results for the PK parameters $\mathrm{AUC}_{0-\mathrm{t}}$ and $\mathrm{C}_{\max }$ at significance levels of $0.05,0.01$, and 0.001 after comparison.

\section{Conclusions}

In conclusion, a rapid and simple LC-MS/MS method for CPPU detection was developed and validated. We then employed this method to investigate the PK characteristics of CPPU in adult and juvenile rats. The $A U C_{0-t}$ and $C_{\max }$ of both adult and juvenile rats treated with CPPU at $10-90 \mathrm{mg} / \mathrm{kg}$ increased in a dose-dependent manner. The $\mathrm{T}_{1 / 2}$ in adult rats was similar to that in juvenile rats. The $\mathrm{AUC}_{0-\mathrm{t}}$ and absolute bioavailability were higher in the latter. These results indicated that juvenile rats were more sensitive to CPPU, which provides an experimental basis for the study of pesticide exposure in children. We hope that the current findings provide insights into the potential safety risks of CPPU, particularly in children.

Author Contributions: Conceptualization, J.W. and D.Z.; methodology; software, B.X. and Q.Z.; validation, Y.H. and L.P.; writing - original draft preparation, L.P., and Q.S.; writing-review and editing, J.W. and D.Z.; funding acquisition, D.Z. All authors have read and agreed to the published version of the manuscript.

Funding: This study was supported by the National Key R\&D Program of China, grant number 2016YFD0401103; and National Natural Science Foundation of China, grant number 81903708. 
Institutional Review Board Statement: The study was approved by the Institutional Animal Care and Use Committee of CDSER of Zhejiang University (IACUC-17-211).

Informed Consent Statement: Not applicable.

Data Availability Statement: The data that support the findings of this study are available on request from the corresponding author. The data are not publicly available due to privacy or ethical restrictions.

Conflicts of Interest: The authors declare no conflict of interest.

Sample Availability: Sample of the compounds are not available from the authors.

\section{References}

1. Orellana-Silla, A.; Armenta, S.; de la Guardia, M.; Mercader, J.V.; Esteve-Turrillas, F.A. Development of immunosorbents for the analysis of CPPU in fruit juices by ion mobility spectrometry. Anal. Bioanal. Chem. 2018, 410, 5961-5967. [CrossRef] [PubMed]

2. Chen, W.; Jiao, B.; Su, X.; Zhao, Q.; Qin, D.; Wang, C. Dissipation and residue of CPPU in citrus fruits. Bull. Environ. Contam. Toxicol. 2013, 90, 756-760. [CrossRef]

3. Shi, H.; Liao, H.; Cui, S.; Luo, Z.; Ma, X. Effects of CPPU on the morphologymetabolite accumulation, and transcriptional responses of Siraitiagrosvenorii Fruit. Molecules 2019, 24, 4076. [CrossRef] [PubMed]

4. Gong, G.; Kam, H.; Tse, Y.; Lee, S.M. Cardiotoxicity of forchlorfenuron(CPPU) in zebrafish (Danio rerio) and H9c2 cardiomyocytes. Chemosphere 2019, 235, 153-162. [CrossRef] [PubMed]

5. Zhang, Z.; Guo, K.; Bai, Y.; Dong, J.; Gao, Z.; Yuan, Y.; Wang, Y.; Liu, L.; Yue, T. Identification, synthesis, and safety assessment of CPPU (1-(2-chloro-4-pyridyl)-3-phenylurea) and its metabolites in kiwifruits. J. Agric. Food Chem. 2015, 63, 3059-3066. [CrossRef] [PubMed]

6. $\quad$ Bu, Q.; Wang, X.; Xie, H.; Zhong, K.; Wu, Y.; Zhang, J.; Wang, Z.; Gao, H.; Huang, Y. 180 Day repeated-dose toxicity study on CPPUin Sprague-Dawley rats and its effects on the production of steroid hormones. J. Agric. Food Chem. 2019, 67, 10207-10213. [CrossRef] [PubMed]

7. Zhu, D.; Ping, L.; Shen, X.; Hong, Y.; Weng, Q.; He, Q.; Wang, J.; Wang, J. Effects of prepubertal exposure to CPPU through prenatal and postnatal gavage administration in developing Sprague-Dawley. Rats. Reprod. Toxicol. 2020, 98, 157-164. [CrossRef] [PubMed]

8. Zhang, J.; Yang, M.; Luan, P.; Jia, W.; Liu, Q.; Ma, Z.; Dang, J.; Lu, H.; Ma, Q.; Wang, Y.; et al. Associations between cytochrome P450 (CYP) gene single-nucleotide polymorphisms and second-to-fourth digit ratio in Chinese university students. Med. Sci. Monit. 2021, 27, e930591. [CrossRef] [PubMed]

9. Li, H.; Lampe, J.N. Neonatal cytochrome P450 CYP3A7: A comprehensive review of its role in development, disease, and xenobiotic metabolism. Arch. Biochem. Biophys. 2019, 673, 108078. [CrossRef] [PubMed]

10. Timchalk, C.; Poet, T.S.; Kousba, A.A. Age-dependent pharmacokinetic and pharmacodynamic response in preweanling rats following oral exposure to the organophosphorus insecticide chlorpyrifos. Toxicology 2006, 220, 13-25. [CrossRef]

11. Vidair, C.A. Age dependence of organophosphate and carbamate neurotoxicity in the postnatal rat: Extrapolation to the human. Toxicol. Appl. Pharmacol. 2004, 196, 287-302. [CrossRef] [PubMed]

12. Warner, A. Drug use in the neonate: Interrelationships of pharmacokinetics, toxicity, and biochemical maturity. Clin. Chem. 1986, 32, 721-727. [CrossRef] [PubMed]

13. Pu, C.-H.; Lin, S.-K.; Chuang, W.-C.; Shyu, T.-H. Modified QuEChERS method for 24 plant growth regulators in grapes using LC-MS/MS. J. Food Drug Anal. 2018, 26, 637-648. [CrossRef] [PubMed]

14. Yan, B.; Guo, W. A novel identification method for CPPU-Treated kiwifruits based on images. J. Sci. Food Agric. 2019, 99, 6234-6240. [CrossRef] [PubMed]

15. Shi, X.M.; Jin, F.; Huang, Y.T.; Du, X.W.; Li, C.M.; Wang, M.; Shao, H.; Jin, M.J.; Wang, J. Simultaneous determination of five plant growth regulators in fruits by modified quick, easy, cheap, effective, rugged, and safe (QuEChERS) extraction and liquid chromatography-tandem mass spectrometry. J. Agric. Food Chem. 2012, 60, 60-65. [CrossRef]

16. Timchalk, C.; Kousba, A.A.; Poet, T.S. An age-dependent physiologically based pharmacokinetic/pharmacodynamic model for the organophosphorus insecticide chlorpyrifos in the preweanling rat. Toxicol. Sci. 2007, 98, 348-365. [CrossRef] [PubMed]

17. Ziegler, E.E.; Edwards, B.B.; Jensen, R.L.; Mahaffey, K.R.; Fomon, S.J. Absorption and retention of lead by infants. Pediatr. Res. 1978, 12, 29-34. [CrossRef] [PubMed]

18. Bruckner, J.V. Differences in sensitivity of children and adults to chemical toxicity: The NAS panel report. Regul. Toxicol. Pharmacol. 2000, 31, 280-285. [CrossRef]

19. US Environmental Protection Agency, Office of Prevention, Pesticides and Toxic Substances. Pesticide Fact Sheet [EB/OL]. U.S.EPA. 2004. Available online: https:/ / www3.epa.gov/pesticides/chem_search/reg_actions/registration/fs_PC-128819_01Sep-04.pdf (accessed on 20 October 2017).

20. Padilla, S.; Corum, D.; Padnos, B.; Hunter, D.; Beam, A.; Houck, K.; Sipes, N.; Kleinstreuer, N.; Knudsen, T.; Dix, D.; et al. Zebrafish developmental screening of the ToxCast ${ }^{\mathrm{TM}}$ Phase I chemical library. Reprod. Toxicol. 2012, 33, 174-187. [CrossRef] [PubMed] 
21. Arena, M.; Auteri, D.; Barmaz, S.; Bellisai, G.; Brancato, A.; Brocca, D.; Bura, L.; Byers, H.; Chiusolo, A.; European Food Safety Authority (EFSA); et al. Peer review of the pesticide risk assessment of the active substance forchlorfenuron. EFSA J. 2017, 15, 4874. [CrossRef]

22. Negre, M.; Passarella, I.; Vindrola, D.; Baglieri, A. Determination of forchlorfenuron in fruits by solid phase or QuECHERSextraction and LC-UV or LC/MS/MS. J. AOAC Int. 2014, 97, 938-941. [CrossRef] [PubMed]

23. Zhang, Z.; Gao, Z.; Wang, Y.; Yuan, Y.; Dong, J.; Yue, T. Transformation products elucidation of forchlorfenuron in postharvest kiwifruit by time-of-flight mass spectrometry. PLoS ONE 2017, 12, e0184021. [CrossRef] [PubMed]

24. Bioanalytical Method Validation Guidance for Industry, U.S. Department of Health and Human Services, Food and Drug Administration, Center for Drug Evaluation and Research (CDER), Center for Veterinary Medicine (CVM). Food and Drug Administration Guidance for Industry: Bioanalytical Method Validation. May 2018. Available online: https://www.fda.gov/ regulatory-information/search-fda-guidance-documents/bioanalytical-method-validation-guidance-industry (accessed on 4 August 2020).

25. Trevor, H. Evaluation of the New Active CPPU in the Product Sitofex 10 EC Plant Growth Regulator. Australian Pesticide and Veterinary Medicines Authority. 2005. Available online: https://apvma.gov.au/node/13791 (accessed on 1 September 2005). 